\title{
2020 The year of your choice between digital and conventional dentistry.
}

Bogdan Oprea ${ }^{1}$

${ }^{1}$ Lucian Blaga University, Sibiu, Romania

We are at the end of 2019, and, as usual, at the end of the year, it is a time of balance and plans for the coming year (2020).

If we look back at what happened professionally, technologically in the year just ended; certainly, an essential aspect worth analyzing is the progress of digital technologies and their increasingly active presence in the various branches of dentistry.

Indeed, we are still on the upward side of the evolution of these technologies, and we cannot even dare to imagine how much and in what direction they will evolve. We can see that a level of maturity of the development of these solutions has been reached, both in terms of hardware and software that allows, today, to create and offer to our patients a complete digital workflow from the first consultation until the end of treatment.

The space allocated for this editorial is far too short to try to make a list of all the already existing and functional technologies that can be implemented in any dental office or clinic.

When analyzing a patient's path from the first consultation to the end of treatment, we can see that at every step, digital technologies are present and play a significant role.

First of all, we have at our disposal extremely robust and developed patient management programs that help us to efficiently manage not only the patient's medical information but also the activity of the office. The maturation of these programs also brought with it the introduction of modules specific to CRM programs that also allow the management of the managerial/financial activity of the firm. The electronic diary is the most powerful tool for managing daily activities based on the principles of efficiency, productivity, and profitability while offering patients a much closer and more friendly relationship with the dental office. We are at a time when we can have a "paperless" office much more organized and efficient than by classical methods. The use of these types of software platforms will facilitate and shorten the "bureaucracy" specific to physical patient files.

Leaving the "bureaucratic" area and entering the clinical part, the first technology I would like to draw your attention to is digital radiology. Of course, we are all already familiar with the use of digital $\mathrm{x}$-rays that have already become "normal" in current practice. But the "normal" that. I propose to focus on in 2020 is $3 \mathrm{D} / \mathrm{CBCT}$ radiology. It is a time of "revolution" in which we should take precedence over scientific publications and accept that the CBCT should become the QUALITY STANDARD for radiological examination at the first consultation (I would like to point out that this is a personal opinion that I assume). The information provided by $2 \mathrm{D}$ radiology is currently insufficient to be able to make a complete/correct diagnosis and to be able to carry out an adequate treatment plan that offers long-term dental health to patients. Although internationally, more and more specialized professional associations recommend the CBCT as the method of investigation of choice in their field of activity, there is still no common, general opinion on this type of investigation. But if we analyze coldly, we can find that a single CBCT investigation at the beginning of treatment will give us all the information we need for diagnosis and treatment. Technological developments have led to better and better performance of these devices and to greater patient safety. There are standard resolution CBCTs in which the radiation dose received by the patient is equivalent to that received during 
a 3-4 hour flight. I believe that the ALARA principles are perfectly respected when we use this type of investigation. So, my first recommendation for you in 2020 is to start using CBCT investigation as much as possible to establish diagnoses and treatment plans during the first consultation.

Now that we have the digital Xray investigation performed, it is time to move on to the clinical examination. Here are two essential digital technologies: digital radiology and intraoral scanner for making the optical impression. The classic clinical examination is irreplaceable, but the two technologies will allow us to examine from perspectives and angles impossible to access in reality. With the help of these two technologies, we can create our "virtual patient" who can be analyzed and consulted indefinitely even after the real patient has left the office. Digital photography, not only is accessible for any doctor (both financially but also in terms of the learning curve), but still, it is a communication tool (both within the team and with the patient) of inestimable value. My second recommendation for 2020: start documenting your entire activity.

Regarding the optical fingerprint and the intraoral scanner, things are straightforward: it is the "must-have" tool that should be at the forefront of your growth and development plan. Virtual models for virtual clinical examination, communication with the digital laboratory, prosthetics, orthodontics, dental aesthetics, surgery/implantology, endodontics are just some of the areas where its usefulness is invaluable. Increased accuracy, time savings, and the comfort of the patient and the medical team are just some of the reasons why you should not miss the opportunity for 2020 to be the year in which this tool becomes part of your therapeutic arsenal.

Chairside CAD-CAM systems are the next technology you should focus on. The possibility of achieving, not only the optical impression but also the fabrication of the various types of prosthetic restorations on natural teeth or implants is a big step forward in terms of changing paradigms in analog dentistry. Medically speaking, chairside CADCAM systems open the door for the most conservative types of preparations. We will prepare strictly the dental tissues that are affected, we will keep the limits of preparations away from periodontium, a classic crown will become the last option, and the chances of preserving the vitality of the teeth will increase when we use these types of restorations. The possibility of introducing the concept of "one session dentistry" will bring multiple benefits (medical, managerial, and financial) for both the patient and the doctor and practice. 3D printers experienced a real explosion in 2019 in terms of their use in dentistry. From quarter to quarter, there is a growing increase in both types of printers and resins with dental indications.

Of course, there are still many exciting and promising digital technologies: computerized occlusion analysis systems, digital facial arches, virtual articulators, online platforms for analysis and planning of dental aesthetics cases, facial scanners, and much more.

What is, however, extremely important is that these technologies are interconnected. They are not just stand-alone devices or software that have particular functions but can communicate with each other and integrate into digital examination or treatment workflows.

Going back to the first consultation, it is obvious that the time the patient spends with us in the office is limited and insufficient to establish all the diagnoses and build an appropriate treatment plan. The possibility of creating a virtual patient using integrated digital technologies (electronic medical record, CBCT, photo documentation, optical fingerprint, digital recordings of occlusion parameters, 3D printed models, etc.) will allow us to carefully and patiently analyze each case without the pressure of time. Digital technology will give us the information and time we need to make the best decisions. The implementation of these digital technologies in daily practice has numerous beneficial medical, epidemiological, managerial/financial effects.

Of course, the major obstacle that most of us see is related to acquisition costs. How do we overcome this obstacle? It's simple and complicated at the same time.

SIMPLE because the financial hurdle is relatively easy to overcome: there are various financing methods, and the ROI (return of 
investment) is extremely fast. SIMPLE because patients will be extremely receptive to these types of treatments and will accept them very quickly once they understand the benefits.

COMPLICATED ...because... US. The blockage is at the level of our attitude. A reset of attitude and mentality is needed. Each of us needs to analyze and decide if he wants his professional future to be digital or not. If the decision is favorable, then you need to keep a permanent focus on the important objectives: "to get the technology!", "to learn about the technolgies", etc.

Each of us needs to analyze and decide whether he wants his professional future to be digital or not. If the decision is positive, then we need a permanent focus on the objective and to find solutions from the obstacles that stand in the way. The most important piece of advice I can give you is: remove "No" and "Yes but..." and enter "Yes" and "I will find the solution". Don't let the defeatist approach stand in the way of your professional growth and development. Always think about the benefits that these technologies will bring you and make these positive thoughts charge you with the energy needed to travel from dream to reality.

It's easy? No. But not impossible. It takes work. But you are used to work, so don't let time pass. Decide that 2020 will be the year of your professional "digitization" and... start to work on it! Attention the work does not end at the time of purchase. Implementation is also essential. It takes work to learn to master these technologies, and it takes work to explain the benefits to each patient.

But as I said.... we are not afraid of work.

If you intend for 2020 to be the year of your professional "digitalization" here are some questions you should answer:

1. Do I need it?

2. What technologies are available?

3. How to start?

4. How to choose?
5. What solutions do I have available to make the purchase?

6. How to integrate (at the office and the patient level).

The first question is essential. If the answer to this question is a positive one, then in the following, you will find support to find the right answers.

And because I don't want to ask you just questions, here are some useful tips on how to "digitize" your practice:

1. Define what kind dentistry you wish to offer to your patients and what technologies you need to do that.

2. Think of the future needs.

3. Decide which technology serves your best present and future needs.

4. Investigate and find the systems that seem to be right for your current and future needs.

5. Compare and analyze but not in terms of acquisition costs.

6. Speak with colleagues who already use this technology.

7. Ask the right questions to the sellers.

8. Ask for an office drive test.

9. Make your Bussines plan.

10.Learn and train before, your system will arrive.

Of course, the choice between the classic analog or the new digital is a personal one for each of you. But one thing is for sure: digital dentistry is not a "fleeting fashion" but is the present and the future.

In conclusion, I offer you for analysis and meditation the following extremely valid thoughts for our profession:

"Resolve to be a master of change rather than a victim of change" - Brian Tracy

"A goal without a plan is just a dream" Antoine de Saint Exupery.

"A dream is just a dream until you make it a goal and set a date" - TD Jakes.

Let's see each other well in a 2020 as digital as possible.

\section{Corresponding author:}

